\title{
THE EXISTENCE OF A CLASS OF KIRKMAN SQUARES OF INDEX 2
}

\author{
E. R. LAMKEN AND S. A. VANSTONE \\ (Received 15 August 1985; revised 20 January 1986) \\ Communicated by L. Caccetta
}

\begin{abstract}
A Kirkman square with index $\lambda$, latinicity $\mu$, block size $k$ and $v$ points, $K S_{k}(v ; \mu, \lambda)$, is a $t \times t$ array $(t=\lambda(v-1) / \mu(k-1))$ defined on a $v$-set $V$ such that (1) each point of $V$ is contained in precisely $\mu$ cells of each row and column, (2) each cell of the array is either empty or contains a $k$-subset of $V$, and (3) the collection of blocks obtained from the nonempty cells of the array is a $(v, k, \lambda)$-BIBD. For $\mu=1$, the existence of a $K S_{k}(v ; \mu, \lambda)$ is equivalent to the existence of a doubly resolvable $(v, k, \lambda)$ BIBD. In this case the only complete results are for $k=2$. The case $k=3, \lambda=1$ appears to be quite difficult although some existence results are available. For $k=3, \lambda=2$ the problem seems to be more tractable. In this paper we prove the existence of a $K S_{3}(v ; 1,2)$ for all $v \equiv 3(\bmod 12)$.
\end{abstract}

1980 Mathematics subject classification (Amer. Math. Soc.): 05 B 30.

\section{Introduction}

A Kirkman square with index $\lambda$, latinicity $\mu$, block size $k$ and $v$ points, $K S_{k}(v ; \mu, \lambda)$, is a $t \times t(t=\lambda(v-1) / \mu(k-1))$ array defined on a $v$-set $V$ such that

(1) each point of $V$ is contained in precisely $\mu$ cells of each row and column,

(2) each cell of the array is either empty or contains a $k$-subset of $V$, and

(3) the collection of blocks obtained from the nonempty cells of the array is a $(v, k, \lambda)$-BIBD.

The existence question for $K S_{2}(v ; \mu, \lambda)$ has been completely settled [5]. For $\mu=1$, the existence of a $K S_{k}(v ; \mu, \lambda)$ is equivalent to the existence of a doubly resolvable $(v, k, \lambda)$-BIBD. A doubly resolvable $(v, k, \lambda)$-BIBD is denoted by

(C) 1988 Australian Mathematical Society $0263-6115 / 88$ \$A2.00 + 0.00 
$D R(v, k, \lambda)$-BIBD. The existence question for $D R(v, k, \lambda)$-BIBDs with $k \geqslant 3$ is open. Of particular interest to us is the case $k=3$. A necessary condition for the existence of a $K S_{3}(v ; 1,1)$ is $v \equiv 3(\bmod 6)$. The best result, thus far, for $K S_{3}(v ; 1,1) s$ is asymptotic.

THEOREM 1.1 [8]. There exists a constant $v_{1}$ such that for all $v \geqslant v_{1}$ and $v \equiv 3$ (mod 6) there exists a $K S_{3}(v ; 1,1)$.

In this paper, we consider the next case $k=3$ and $\lambda=2 . K S_{3}(v ; 1,2) s$ are equivalent to $D R(v, 3,2)$-BIBDs and have been called doubly resolvable twofold triple systems of order $v$ (DRTTS $(v)$ ) ([1]). A necessary condition for the existence of a $K S_{3}(v ; 1,2)$ is $v \equiv 0(\bmod 3)$. A $K S_{3}(3 ; 1,2)$ defined on $\{\infty, 0,1\}$ is

\begin{tabular}{|l|l|}
\hline$\infty 01$ & \\
\hline & $\infty 01$ \\
\hline
\end{tabular}

It is known that there do not exist $K S_{3}(6 ; 1,2)$ and $K S_{3}(9 ; 1,2)[6]$. The next smallest design has recently been constructed. A $K S_{3}(12 ; 1,2)$ appears in [4]. $K S_{3}(v ; 1,2)$ s are also known to exist for $v=15,18,21,24,27,30$ and 33. These designs were constructed using starters and adders ([1], for $v=33$, Lemma 3.6). In the next section, we give some recursive constructions for $K S_{3}(v ; 1,2)$ s. In the last section, we apply these constructions to prove the existence of $K S_{3}(v ; 1,2) \mathrm{s}$ for $v \equiv 3(\bmod 12)$.

\section{Constructions}

Let $V$ be a set of $v$ elements. Let $G_{1}, G_{2}, \ldots, G_{m}$ be a partition of $V$ into $m$ sets. A $\left\{G_{1}, G_{2}, \ldots, G_{m}\right\}$-frame $F$ with block size $k$, index $\lambda$ and latinicity $\mu$ is a square array of side $v$ which satisfies the properties listed below. We index the rows and columns of $F$ by the elements of $V$.

(1) Each cell is either empty or contains a $k$-subset of $V$.

(2) Let $F_{i}$ be the subsquare of $F$ indexed by the elements of $G_{i} . F_{i}$ is empty for $i=1,2, \ldots, m$.

(3) Let $j \in G_{i}$. Row $j$ of $F$ contains each element of $V-G_{i} \mu$ times and column $j$ of $F$ contains each element of $V-G_{i} \mu$ times.

(4) The collection of blocks obtained from the nonempty cells of $F$ is a $G D D\left(v ; k ; G_{1} G_{2}, \ldots, G_{m} ; 0, \lambda\right)$ (see [14] for $G D D$ notation).

If $\left|G_{i}\right|=h$ for $i=1,2, \ldots, m$, we call $F$ a $(\mu, \lambda ; k, m, h)$-frame. 
We will use frames to provide some product constructions for $K S_{3}(v ; 1,2) \mathrm{s}$. The first result uses a $(1,2,3 ; m, 1)$-frame.

THEOREM 2.1. If there exists a $(1,2 ; 3, m, 1)$-frame, $a K S_{3}(n+1 ; 1,2)$ and three mutually orthogonal Latin squares of side $n$, then there is a $K S_{3}(m n+1 ; 1,2)$ which contains as a subarray a $K S_{3}(n+1 ; 1,2)$.

Proof. Let $V=\{1,2, \ldots, n\}$ and let $V_{i}=V \times\{i\}$ for $i=1,2, \ldots, m$. Let $L_{1}$, $L_{2}$ and $L_{3}$ be a set of three mutually orthogonal Latin squares of side $n$ defined on $V . L$ will denote the array of triples formed by the superposition of $L_{1}, L_{2}$ and $L_{3} . L_{i j k}$ is the $n \times n$ array of triples formed by replacing each triple $(a, b, c)$ in $L$ with the triple $\left(a_{i}, b_{j}, c_{k}\right)$ where $a_{i} \in V_{i}, b_{j} \in V_{j}$ and $c_{k} \in V_{k}$.

Let $K_{i}$ be a $K S_{3}(n+1 ; 1,2)$ defined on $V_{i} \cup\{\infty\}$. Let $F$ be a $(1,2 ; 3, m, 1)$ frame defined on $\{1,2, \ldots, m\}$ such that $i$ is missing from cell $(i, i)$ for $i=$ $1,2, \ldots, m$.

We construct a $K S_{3}(m n+1 ; 1,2)$ on $(V \times\{1,2, \ldots, m\}) \cup\{\infty\}$ as follows. Replace each triple $(i, j, k)$ in $F$ with the $n \times n$ array $L_{i j k}$. In each cell $(i, i)$ of $F$, place the $n \times n$ array $K_{i}$ for $i=1,2, \ldots, m$. The resulting array $A$ has size $m n \times m n$. Each distinct pair in $(V \times\{1,2, \ldots, m\}) \cup\{\infty\}$ occurs twice in $A$. Each element in $(V \times\{1,2, \ldots, m\}$ occurs once in each row and each column of $A$. Thus, $A$ is a $K S_{3}(m n+1 ; 1,2)$.

The next result will be used for $(1,2 ; 3, m, h)$-frames with $h=1,3$ and 6 . This construction also appears in [2].

THEOREM 2.2. If there exists a $(1,2 ; 3, m, h)$-frame, a $K S_{3}(h n+w ; 1,2)$ which contains as a subarray a $K S_{3}(w ; 1,2)(w \geqslant 3)$ and three mutually orthogonal Latin squares of side $n$, then there is a $K S_{3}(h m n+w ; 1,2)$ which contains as a subarray $a$ $K S_{3}(w ; 1,2)$.

Proof. Let $V=\left\{x_{1}^{i}, x_{2}^{i}, \ldots, x_{h}^{i} \mid 1 \leqslant i \leqslant m\right\}$ and let $G_{i}=\left\{x_{1}^{i}, x_{2}^{i}, \ldots, x_{h}^{i}\right\}$ for $i=1,2, \ldots, m$. Let $W=\left\{\infty_{1}, \infty_{2}, \ldots, \infty_{w}\right\}$ and let $N=\{1,2, \ldots, n\}$.

Let $L_{1}, L_{2}$ and $L_{3}$ be a set of three mutually orthogonal Latin squares of side $n$ defined on $N$. $L$ will denote the array of triples formed by the superposition of $L_{1}, L_{2}$ and $L_{3} . L_{i j k}$ is the $n \times n$ array of triples formed by replacing each triple $(a, b, c)$ in $L$ with the triple $\left(a_{i}, b_{j}, c_{k}\right)$ where $a_{i} \in N \times\{i\}, b_{j} \in N \times\{j\}$ and $c_{k} \in N \times\{k\}$.

Let $F$ be a $(1,2,3 ; m, h)$-frame defined on $V . F$ is a $\left\{G_{1}, G_{2}, \ldots, G_{m}\right\}$-frame. Construct an $h m n \times h m n$ array $H$ from $F$ by replacing each triple $(x, y, z)$ in $F$ with the $n \times n$ array $L_{x y z}$. $H$ contains a diagonal of $m h n \times h n$ empty arrays. 
Let $K_{i}$ denote a $K S_{3}(h n+w ; 1,2)$ defined on $\left(N \times G_{i}\right) \cup W$ which contains as a subarray a $K S_{3}(w ; 1,2)$ defined on $W$. Let $A$ denote the subarray defined on $W . K_{i}$ can be partitioned as follows.

$$
\left.K_{i}=\begin{array}{|l|l|}
\hline A & B_{i} \\
\hline C_{i} & D_{i} \\
\hline
\end{array}\right\} \begin{aligned}
& \} w n-1 \\
&
\end{aligned}
$$

where $A$ and $D_{i}$ are square arrays of side $w-1$ and $h$ respectively.

We now construct a new array $K$ from $H$ and the $K_{i}$ 's for $i=1,2, \ldots, m . K$ is defined on $(N \times V) \cup W$.

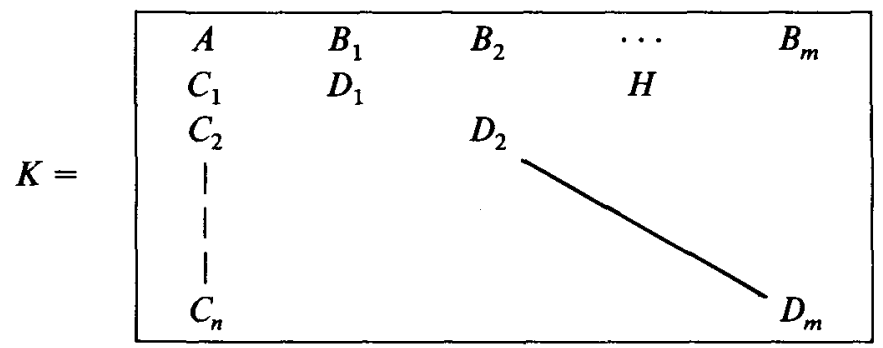

$K$ is a square array of side $h n m+w-1$. Each element of $(N \times V) \cup W$ occurs precisely once in each row and each column of $K$. Every distinct pair in $(N \times V) \cup W$ occurs twice in $K$. Thus, $K$ is a $K S_{3}(h m n+w ; 1,2)$ which contains as a subarray a $K S_{3}(w ; 1,2)(A)$.

The last construction in this section is an indirect product for $K S_{3}(v ; 1,2) s$. Before describing the construction, we recall the definition of an $\operatorname{IA}(n, k, s)$. Let $V$ be a finite set of size $n$. Let $K$ be a subset of size $k$ of $V$. An incomplete orthogonal array $I A(n, k, s)$ is an $n^{2}-k^{2} \times s$ array written on the symbol set $V$ such that every ordered pair of symbols in $V \times V-(K \times K)$ occurs in any ordered pair of columns from the array. We may think of an $I A(n, k, s)$ as a set of $s-2$ mutually orthogonal Latin squares of order $n$ which are missing a subsquare of order $k$. We need not be able to fill in the $k \times k$ missing subsquares with Latin squares of side $k$.

THEOREM 2.3. Let $u, v$ and $w$ be non-negative integers such that $0 \leqslant u<w<v$. Suppose that $v-u \equiv 0(\bmod h)$ and $w-u \equiv 0(\bmod h)$. If there exists $a$ $(1,2,3 ; m, h)$-frame, and $I A((v-u) / h,(w-u) / h, 5), a K S_{3}(v+1 ; 1,2)$ which contains as a subarray a $K S_{3}(w+1 ; 1,2)$, and a $K S_{3}(m(w-u)+u+1 ; 1,2)$, then there exists a $K S_{3}(m(v-u)+u+1 ; 1,2)$.

Proof. Let $V=\left\{x_{1}^{i}, x_{2}^{i}, \ldots, x_{h}^{i} \mid 1 \leqslant i \leqslant m\right\}, W=\{1,2, \ldots,(v-u) / y h\}, W_{1}$ $=\{1,2, \ldots,(w-u) / h\}$ and $U=\left\{\infty_{1}, \infty_{2}, \ldots, \infty_{u+1}\right\}$. Let $G_{i}=$ $\left\{x_{1}^{i}, x_{2}^{i}, \ldots, x_{h}^{i}\right\}$. 
Let $F$ be a $(1,2 ; 3, m, h)$-frame defined on $V$ such that $F$ is a $\left\{G_{1}, G_{2}, \ldots, G_{m}\right\}$-frame.

We construct a set of three mutually orthogonal Latin squares of order $(v-u) / h$ defined on $W$ which are missing subsquares of order $(w-u) / h$ defined on $W_{1}$ in the upper left hand corners of the arrays from the $I A((v-u) / h,(w-u) / h, 5)$. Let $I$ be the $(v-u) / h \times(v-u) / h$ array of triples formed from the superposition of these three squares. The array $I_{i j k}$ will be the array of triples formed by replacing each triple $(a, b, c)$ in $I$ with the triple $\left(a_{i}, b_{j}, c_{k}\right)$ where $a_{i} \in W \times\{i\}, b_{j} \in W \times\{j\}$ and $c_{k} \in W \times\{k\}$.

Next we construct an $m(v-u) \times m(v-u)$ array from $F$ by replacing each triple $(i, j, k)$ in $F$ by the $(v-u) / h \times(v-u) / h$ array $I_{i j k}$. (Empty cells in $F$ are replaced by $(v-u) / h \times(v-u) / h$ empty arrays.) Call the resulting array $H^{\prime} . H^{\prime}$ contains a diagonal of $m(v-u) \times(v-u)$ empty arrays. We can partition $H^{\prime}$ into $m^{2}(v-u) \times(v-u)$ arrays. Denote these subarrays by $H_{i j}^{\prime}$ for $i, j=1,2, \ldots, m$. We can permute the rows and columns of $H^{\prime}$ so that each subarray $H_{i j}^{\prime}$ contains an empty $(w-u) \times(w-u)$ array in the upper left hand corner. Call this array $H . H$ also contains a diagonal of $m v-u \times v-u$ empty arrays. $H$ is defined on $W \times V$.

Let $A_{i}$ be a $K S_{3}(v+1 ; 1,2)$ on $\left(W \times G_{i}\right) \cup U$ such that the subarray $K S_{3}(w+1 ; 1,2)$ is defined on $\left(W_{1} \times G_{i}\right) \cup U$. We can partition $A_{i}$ as follows.

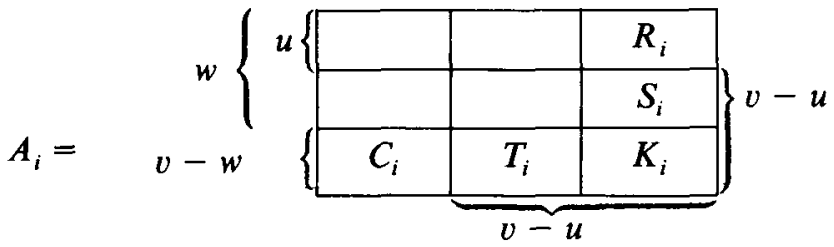

We now construct a square array of side $m(v-u)+u$ using the $A_{i}$ and $H$. This array will be called $B_{1}$ and has the following form.

\begin{tabular}{|c|c|c|cc|c|cc|}
\hline$E$ & $E$ & $R_{1}$ & $E$ & $R_{2}$ & $E$ & $E$ & $R_{m}$ \\
\hline$E$ & $E$ & $S_{1}$ & $E$ & & $E$ & $E$ & \\
\hline$C_{1}$ & $T_{1}$ & $K_{1}$ & & & & & $H$ \\
\hline$E$ & $E$ & & $E$ & $S_{2}$ & & & \\
$C_{2}$ & & & $T_{2}$ & $K_{2}$ & & & \\
\hline & & & & & - & & \\
\hline$E$ & $E$ & & & & & $E$ & $S_{m}$ \\
$C_{m}$ & & & & & & $T_{m}$ & $K_{m}$ \\
\hline
\end{tabular}


The arrays labelled $E$ in $B_{1}$ are empty. They form an $m(w-u)+u \times$ $m(u-u)+u$ array. Place a $K S_{3}(m(w-u)+u+1 ; 1,2)$ defined on $\left(W_{1} \times V\right)$ $\cup U$ in this array. The resulting array $B$ is a $K S_{3}(m(v-u)+u+1 ; 1,2)$ on $(W \times V) \cup U$. Every pair of distinct elements in $(W \times V) \cup U$ occurs precisely twice in $B$ since $F$ and the Kirkman squares used to construct $B$ had index $\lambda=2$. It can be verified that each element in $(W \times V) \cup U$ occurs once in each row and each column of $B$.

\section{Applications}

In order to apply the constructions from the previous section, we will need the following results on frames from [2].

THEOREM 3.1. [2] There exist $(1,2 ; 3, m, 3)$-frames for $m \geqslant 5$ except possibly for $m \in\{6,10,14,16,18,20,22,24,26,28,30,32,34,38,39,42,43,44,46,47,48$, $51,52,59,118,123\}$.

THEOREM 3.2. [2] There exist $(1,2 ; 3, m, 6)$-frames for $m \geqslant 5$ except possibly for $m \in\{10,11,14,15,17,18,19,20,23,24,27,28,32,34,39\}$.

We note that one more value can be deleted from the list of exceptions to Theorem 3.1.

Lemma 3.3. There exists $a(1,2 ; 3,48,3)$-frame.

Proof. Apply the frame singular direct product [2] using a $(1,2 ; 3,6,6)$-frame, three mutually orthogonal Latin squares of side 4 and a $(1,2 ; 3,8,3)$-frame.

The constructions also require the existence of some $K S_{3}(v ; 1,2)$ s which contain as subarrays $K S_{3}(w ; 1,2)$ s where $w \geqslant 3$.

LEMMA 3.4. There exists a $K S_{3}(v ; 1,2)$ which contains as a subarray a $K S_{3}(3 ; 1,2)$ for $v=15,21,27,39,51,63$ and 81. Furthermore, there exists a $K S_{3}(63 ; 1,2)$ which contains as a subarray a $K S_{3}(15 ; 1,2)$.

Proof. A $K S_{3}(15 ; 1,2)$ is displayed in Figure 1. A starter and adder for a $K S_{3}(21 ; 1,2)$ are listed in $[1]$. Since there exist $K S_{3}(v ; 1,1)$ for $v=27,39,51,63$ and 81 ([3], [9], [11],[12]), there exists $K S_{3}(v ; 1,2)$ which contain as subarrays $K S_{3}(3 ; 1,2)$ for $v=27,39,51,63$ and 81 . To construct a $K S_{3}(63 ; 1,2)$ which contains as a subarray a $K S_{3}(15 ; 1,2)$, we apply Theorem 2.2 using a $(1,2 ; 3,5,3)$-frame, a $K S_{3}(15 ; 1,2)$ which contains a $K S_{3}(3 ; 1,2)$ and 3 mutually orthogonal Latin squares of side 4 . 


\begin{tabular}{|l|l|l|l|l|l|l|l|l|l|l|l|l|l|}
\hline$x 0 \overline{0}$ & $2 \overline{4} \overline{5}$ & $4 \overline{2} \overline{1}$ & & $1 \overline{\overline{6}} \overline{2}$ & & & 356 & & & & & & \\
\hline & $x \overline{1} \overline{1}$ & $3 \overline{5} \overline{6}$ & $5 \overline{4} \overline{2}$ & & $2 \overline{0} \overline{2}$ & & & 460 & & & & & \\
\hline & & $x 2 \overline{2}$ & $4 \overline{6} \overline{0}$ & $6 \overline{5} \overline{2}$ & & $2 \overline{1} \overline{4}$ & & & 501 & & & & \\
\hline $4 \overline{2} \overline{5}$ & & & $x 2 \overline{2}$ & $5 \overline{0} \overline{\overline{1}}$ & $0 \overline{6} \overline{4}$ & & & & & 612 & & & \\
\hline & $5 \overline{2} \overline{6}$ & & & $x 4 \overline{4}$ & $6 \overline{1} \overline{2}$ & $1 \overline{0} \overline{5}$ & & & & & $02 \overline{3}$ & & \\
\hline $2 \overline{1} \overline{6}$ & & $6 \overline{4} \overline{0}$ & & & $x 5 \overline{5}$ & $0 \overline{2} \overline{3}$ & & & & & & 134 & \\
\hline $1 \overline{2} \overline{4}$ & $3 \overline{2} \overline{0}$ & & $0 \overline{5} \overline{1}$ & & & $x 6 \overline{6}$ & & & & & & & 245 \\
\hline $35 \overline{6}$ & & & & & & & $x 0 \overline{0}$ & $2 \overline{4} \overline{5}$ & $4 \overline{3} \overline{1}$ & & $1 \overline{6} \overline{2}$ & & \\
\hline & 460 & & & & & & & $x 1 \overline{1}$ & $3 \overline{5} \overline{6}$ & $5 \overline{4} \overline{2}$ & & $2 \overline{0} \overline{3}$ & \\
\hline & & 501 & & & & & & & $x 2 \overline{2}$ & $4 \overline{6} \overline{0}$ & $6 \overline{5} \overline{3}$ & & $2 \overline{1} \overline{4}$ \\
\hline & & & 612 & & & & $4 \overline{2} \overline{5}$ & & & $x 3 \overline{2}$ & $5 \overline{0} \overline{1}$ & $0 \overline{6} \overline{4}$ & \\
\hline & & & & 023 & & & & $5 \overline{3} \overline{6}$ & & & $x 4 \overline{4}$ & $6 \overline{1} \overline{2}$ & $1 \overline{0} \overline{5}$ \\
\hline & & & & & 1324 & & $2 \overline{1} \overline{6}$ & & $6 \overline{4} \overline{0}$ & & & $x 5 \overline{5}$ & $0 \overline{2} \overline{3}$ \\
\hline & & & & & & 245 & $1 \overline{\overline{3}} \overline{4}$ & $3 \overline{2} \overline{0}$ & & $0 \overline{5} \overline{1}$ & & & $x 6 \overline{6}$ \\
\hline
\end{tabular}

Figure 1.

A $K S_{3}(15 ; 1,2)$ which contains a $K S_{3}(3 ; 1,2)$.

Finally, we require three designs which we constructed directly using starters and adders and the following result. For definitions and results on 1-rotational $(v, 3,1)$-BIBDs, see [7].

Lemma 3.5. Let $k=(v-3) / 6$. Let $\left(B_{0}, B_{1}, \ldots, B_{k}\right)$ be a starter for a 1rotational $(v, 3,1)$-BIBD defined on $Z_{v-1} \cup\{\infty\}$. Let $A=\left(a_{0}, a_{1}, \ldots, a_{k}\right)$ be an adder for $S$. Suppose $S$ and $A$ have the following properties.

(1) $B_{0}=\{\infty, 0,(v-1) / 2\}$ and $a_{0}=0$.

(2) If $b \in B_{i}$ for some $i, 1 \leqslant i \leqslant k$, then $-b \notin B_{j}$ for $j=0,1, \ldots, k$.

(3) For $i=1,2, \ldots, k, a_{i} \neq 0$ or $(v-1) / 2$.

(4) $a_{i}+a_{j} \equiv \equiv 0(\bmod v-1)$ for $1 \leqslant i, j \leqslant k$.

Then there exists a $K S_{3}(v ; 1,2)$.

Proof. If $B_{i}=\{x, y, z\}$, define $-B_{i}=\{-x,-y,-z\}=(v-1-x, v-1-$ $y, v-1-z\}$. A starter for a $K S_{3}(v ; 1,2)$ is $S \cup\left\{-B_{1},-B_{2}, \ldots,-B_{k}\right\}$ and a corresponding adder is $A \cup\left\{-a_{1},-a_{2}, \ldots,-a_{k}\right\}$.

It is known that 1-rotational $(v, 3,1)$-BIBDs exist if and only if $v \equiv 3$ or 9 $(\bmod 24),[7]$.

LEMma 3.6. There exist $K S_{3}(v ; 1,2)$ for $v=33,57$ and 75 .

PRoof. In Table 3, we list the starters and adders required to apply Lemma 3.5. 
We are now in a position to prove our main result.

THEOREM 3.7. There exist a $K S_{3}(v ; 1,2)$ which contains a subarray $K S_{3}(3 ; 1,2)$ for $v \equiv 3(\bmod 12)$.

TABLE 3

Starters and adders for $K S_{3}(v ; 1,2)$ for $v=33,57$ and 75

$\begin{array}{ccccccc}v=33 & & & & & & \\ \text { Starter } & \infty 016 & 128 & 7921 & 3614 & 151928 & 51020 \\ \text { Adder } & 0 & 4 & 22 & 1 & 27 & 14 \\ v=57 & & & & & & \\ \text { Starter } & \infty 028 & 1214 & 3527 & 4722 & 444813 & 303546 \\ \text { Adder } & 0 & 1 & 2 & 13 & 42 & 35 \\ & & 192545 & 334050 & 91736 & 152438 & \\ v=75 & & 19 & 12 & 7 & 51 & \\ \text { Starter } & \infty 037 & 1217 & 3534 & 4724 & 61038 & 81331 \\ \text { Adder } & & 1 & 2 & 4 & 10 & 11 \\ & & 162249 & 445165 & 546214 & 334255 & 182853 \\ & & 35 & 8 & 47 & 7 & 52 \\ & & 152645 & 273963 & & & \end{array}$

Proof. Let $v=12 m+3$. By Lemma 3.4, there exist $K S_{3}(12 m+3 ; 1,2)$ for $m=0,1,2,3$ and 4 . All of these arrays contain a $K S_{3}(3 ; 1,2)$ as a subarray.

Let $N_{1}=\{10,14,16,18,22,24,26,30,34,38,42,46\}, N_{2}=\{24,39,51,123\}, N_{3}$ $=\{20,28,32,44,52\}$ and $N_{4}=\{6,43,47,59,118\}$. Let $N=\bigcup_{i=1}^{4} N_{i}$.

Since there exist $(1,2 ; 3, m, 3$ )-frames for $m \geqslant 5, m \notin N$ (Theorem 3.1, Lemma 3.3), we can apply Theorem 2.2. We first use it with $h=3, w=3$ and $n=4$. Since there exist three mutually orthogonal Latin squares of side 4 and a $K S_{3}(15 ; 1,2)$ with a $K S_{3}(3 ; 1,2)$ as a subarray, there exist $K S_{3}(12 m+3 ; 1,2)$ for $m \geqslant 5$ and $m \notin N$.

Since there exists a $K S_{3}(27 ; 1,2)$ with a $K S_{3}(3 ; 1,2)$ as a subarray and three mutually orthogonal Latin squares of side 8 , we apply Theorem 2.2 with $h=3$, $w=3$ and $n=8$ to construct $K S_{3}(24 m+3 ; 1,2)$ for $m \geqslant 5, m \notin N$. This will construct $K S_{3}(12 m+3 ; 1,2)$ for $m \in N_{1}$. Similarly, we can apply Theorem 2.2 with $h=3, w=3$ and $n=12$ to construct $K S_{3}(36 m+3 ; 1,2)$ for $m \geqslant 5$, $m \notin N$. This will construct $K S_{3}(12 m+3 ; 1,2)$ for $m \in N_{2}$. Applying Theorem 2.2 again with $h=w=3$ and $n=16$ will construct $K S_{3}(48 m+3 ; 1,2)$ for $m \geqslant 5, m \notin N$. This will provide $K S_{3}(12 m+3 ; 1,2)$ for $m \in N_{3}$.

There are now five values of $m$ left to consider, $m \in N_{4}=\{6,43,47,59,118\}$. By Lemma 3.6, there exists a $K S_{3}(12 \cdot 6+3 ; 1,2)$. We construct a $K S_{3}(12 \cdot 43+3 ; 1,2)$ by applying Theorem 2.1 with $m=37$ and $n=14$ since $12 \cdot 43+3=37 \cdot 14+1$. (A $(1,2 ; 3,37,1)$-frame is constructed in [13].) A 
$K S_{3}(12 \cdot 118+3 ; 1,2)$ can be constructed by applying Theorem 2.2 with $m=59$, $h=6, w=3$ and $n=4$.

We use the indirect product (Theorem 2.3) for the two remaining values of $m$. There exist a $(1,2 ; 3,10,1)$-frame [1], a $K S(63 ; 1,2)$ which contains as a subarray a $K S_{3}(87 ; 1,2)$ and an $I A(56,8,5)$. By applying Theorem 2.3 with the parameters $v=62, w=14, u=6, h=1$ and $m=10$, we construct a $K S_{3}(12 \cdot 47+3 ; 1,2)$. Since there exists a $(1,2 ; 3,13,6)$-frame (Theorem 3.2$)$, a $K S_{3}(63 ; 1,2)$ which contains a $K S_{3}(15 ; 1,2)$, a $K S_{3}(87 ; 1,2)$ and an $I A(9,1,5)$, we can apply Theorem 2.3 again with $m=13, h=6, v=62, w=14$ and $u=8$ to construct a $K S_{3}(13(54)+9 ; 1,2)$. This is a $K S_{3}(12 \cdot 59+3 ; 1,2)$.

Note that each of the arrays that we have constructed contains as a subarray a $K S_{3}(3 ; 1,2)$.

\section{References}

[1] C. J. Colbourn and S. A. Vanstone, 'Doubly resolvable twofold triple systems', Congress. Numer. 34 (1982), 219-223.

[2] C. J. Colbourn, K. E. Manson and W. D. Wallis, 'Frames for twofold triple systems', Ars Combin. 17 (1984), 69-78.

[3] R. Fuji-Hara and S. A. Vanstone, 'On the spectrum of doubly resolvable designs', Congress. Numer. 28 (1980), 399-407.

[4] P. Gibbons and R. Mathon, 'Construction methods for Bhaskar Rao and related designs', $J$. Austral. Math. Soc. (to appear).

[5] E. R. Lamken, Coverings, orthogonally resolvable designs and related combinatorial configurations (Ph. D. Thesis, Univ. of Michigan, 1983).

[6] E. J. Morgan, 'Some small quasi-multiple designs', Ars Combin. 3 (1977), 233-250.

[7] K. T. Phelps and A. Rosa, 'Steiner triple systems with rotational automorphisms', Discrete Math. 33 (1981), 57-66.

[8] A. Rosa and S. A. Vanstone, 'Starter-adder techniques for Kirkman squares and Kirkman cubes of small sides', Ars Combin. 14 (1982), 199-212.

[9] A. Rosa and S. A. Vanstone, 'On the existence of strong Kirkman cubes of order 39 and block size 3,' Ann. Discrete Math. 26 (1983), 309-320.

[10] M. Skolem, 'On certain distributions of integers in pairs with given differences', Math. Scand. 5 (1957), 57-68.

[11] D. R. Stinson and S. A. Vanstone, 'A Kirkman square of order 51 and block size 3', Discrete Math. 55 (1985), 107-111.

[12] D. R. Stinson and S. A. Vanstone, 'Orthogonal packings in $P G(5,2)$ ', Aequationes Math. 31 (1986), 159-168.

[13] S. A. Vanstone, 'On mutually orthogonal resolutions and near resolutions', Ann. Discrete Math. 15 (1982), 357-369.

[14] S. A. Vanstone, 'Doubly resolvable designs', Discrete Math. 29 (1980), 77-86.

Department of Combinatorics and Optimization

University of Waterloo

Waterloo, Ontario N21 3G1

Canada 